\title{
TOURISMUS ALS WACHSENDER WIRTSCHAFTSZWEIG UND SEINE BEDEUIUNG FÜR DAS ERLERNEN DER FREMDSPRACHEN
}

D er Trend zu Reisen ins Ausland hält unvermindert an. Zwischen 1980 und heute sind die Einnahmen aus dem internationalen Tourismus rascher angestiegen als diejenigen aus dem Waren- und Dienstleistungsverkehr. Die "World Tourism Organisation" rechnet bis zum Jahr 2020 mit 1,6 Mrd. internationalen Touristenankünften. 1997 wurden weltweit 613 Mio touristische Ankünfte registriert, was einen neuen Rekord darstellt; entsprechend stiegen auch die Reiseausgaben.

In Deutschland ist die Tourismuswirtschaft eine der führenden Branchen. Mit einem Umsatz von 200 Mrd. DM hat sie 1997 die Bauwirtschaft überholt. Insgesamt arbeiten circa 2 Mio. Menschen in der Bundesrepublik in der Tourismusbranche. Noch vor den USA und Japan ist Deutschland das reisefreudigste Land. Dabei nimmt der Anteil der organisierten Urlaubsreisen (Pauschatreisen, Städtereisen) besonders deutlich zu. 78 von 100 Bundesbürgern waren 1997 mindestens einmal im Jahr unterwegs.

Wachsende Mobilität sowie verbesserte Kommunikationsmöglichkeiten (z.B. Reservierungssysteme) sind

\begin{tabular}{|c|c|c|c|}
\hline $\begin{array}{l}\text { Urlaub und Reisen ISY } \\
\text { Ausgewählte Auslandsr }\end{array}$ & eziele der & 1994( & \\
\hline & Gesamt & West & Ost \\
\hline Reisen in Millionen & 67,2 & 53,9 & 13,4 \\
\hline Spanien & 8,1 & $\overline{7,2}$ & 0,9 \\
\hline Außereurop. Lander & 7,2 & 6,3 & 0,9 \\
\hline Italien & 6 & 5,3 & 0,7 \\
\hline Österreich & 5 & 4 & 1 \\
\hline Osteurop. Länder & 3,3 & 2 & 1,3 \\
\hline Frankreich & 3 & 2.6 & 0,3 \\
\hline Griechenland & 2,4 & 1,9 & 0,5 \\
\hline Niederlande & 1,6 & 1,3 & 0,3 \\
\hline Schweiz & 1,5 & 1,2 & 0,2 \\
\hline Dånemark & 1,5 & 1,2 & 0,4 \\
\hline Skandinavien & 1,1 & 0,8 & 0,3 \\
\hline Großbrit./ Irland & 1 & 0,8 & 0,2 \\
\hline
\end{tabular}

\section{María Rosario Martí} Marco

\section{Dpto.Filologías integradas}

Filología Alemana

Universidad de Alicante anspruchsvoller. Die Fernreiselust der Deutschen macht sich auch in der Zahlungsbilanz bemerkbar. Wie bereits 1996, gaben

Seit der Wiedervereiningung hat die Bundesrepublik Deutschland an touristischem Wert gewonnen (Tabelle 1). Sie liegt bei Messereisen weltweit immer noch auf Platz eins, bei Kongreß- und Veranstaltungsreisen sowie als Reiseland generell auf Platz vier. Zur gezielten Förderung des Wirtschaftszweigs hat sich 1996 die Deutsche Tourismus Marketing GmbH konstituiert, die von der Deutschen Zentrale für Tourismus, dem Deutschen Fremdenverkehrsverband, dem Bundesverband der Deutschen Tourismuswitschaft sowie der Deutschen Bahn AG getragen wird. klare Kennzeichen einer zunehmenden Globalisierung. Sinkende Preise führen zu größeren Distanzen bei Flugreisen. Häufigeres Reisen macht zudem den Verbraucher reiseerfahren und damit
Bei den ausländischen Reisezielen zeigt sich immer noch sehr deutlich die unterschiedliche Reiseerfahrung und -historie, die sich natürlich auch in der Wahl ausländischer Reiseziele niederschlägt. Bei den Deutschen bestätigt sich die in den letzten Jahren bekannte Rangreihe mit Spanien auf dem 1. Platz (Tabelle 2), gefolgt von Italien, Österreich, Frankreich und Griechenland als den 5 beliebtesten europäischen Urlaubsländern. 


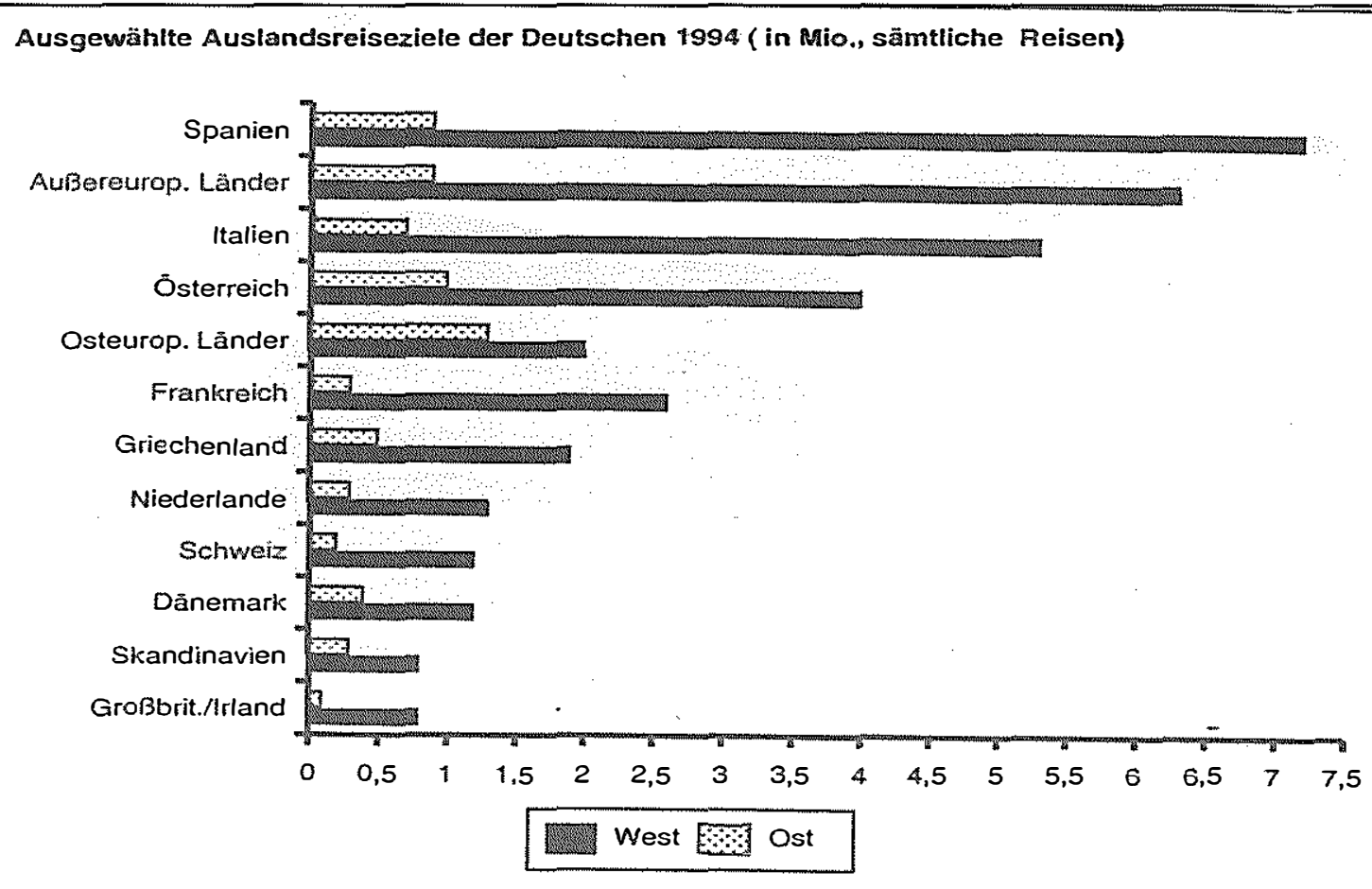

Quelle: U.R 95, Basis-Tab. S. 5-7 (sämtI. Reisen)

Spanien steht seit Jahren mit an der Spitze der beliebtesten Auslandsreiseziele der Deutschen (Tabelle 2). Sowohl das Festland als auch die Inseln besitzen einen ausgesprochen guten Ruf als Reiseziel bei den Deutschen. Spanien wird im allgemeinen Natürlichkeit und Weltoffenheit, aber auch Traditionsbewußtsein und Geschichtsträchtigkeit bescheinigt.

Die Deutschen schätzen in Spanien die Sonne und Wärme, die guten Bademöglichkeiten, sowie die schöne Landschaft. Aber auch die zahlreichen Sehenswürdigkeiten und ganz besonders die Gastíreundschaft der Bevölkerung stehen weit oben auf der Liste der Vorteile Spaniens als Urlaubsland (Tabelle 4).

Was den Touristen im allgemeinen am meisten stört, sind oftmals die anderen Touristen. Dies gilt auch für Spanien. Dort wird der Massentourismus mit seinen Folgen als störend empfunden: verbaute Landschaft, Betonburgen, zuviel Straßenverkehr in den Orten, aber auch Unsicherheit. Besonders moniert wurden dabei

\begin{tabular}{|c|c|c|c|}
\hline \multicolumn{4}{|c|}{ ESPANA } \\
\hline PAIS & 1993 & 1994 & 1995 \\
\hline Francia & 12.070 .214 & 13.696 .012 & 20.289 .355 \\
\hline Portugal & 11.358 .655 & 10.249 .070 & \\
\hline Alemania & 8.713 .281 & 9.678 .673 & 10.446 .756 \\
\hline Reino Unido & 7.485 .129 & 9.170 .041 & \\
\hline Paises Bajos & 2.062 .024 & 2.271 .429 & 2.226 .483 \\
\hline Italia & 2.018 .506 & 2.818 .988 & 3.686 .206 \\
\hline
\end{tabular}

Umwelt- und Abfallprobleme an Stränden und im Wasser (Tabelle 4).

Aus den dargestellten derzeitigen Gegebenheiten erklärt sich ein verstärkter Umgang mit Fremdsprachen und dies nicht nur im Tourismusbereich.

In Deutschland geben rund $75 \%$ der bei den Industrie- und Handelskammern angesiedelten Betriebe und rund $22 \%$ der Handwerksbetriebe Fremdsprachenbedarf an. Je größer ein Betrieb, umso wichtiger werden Fremdsprachen. 


\section{Estancia media de los turistas por pais de residencia según via de acceso}

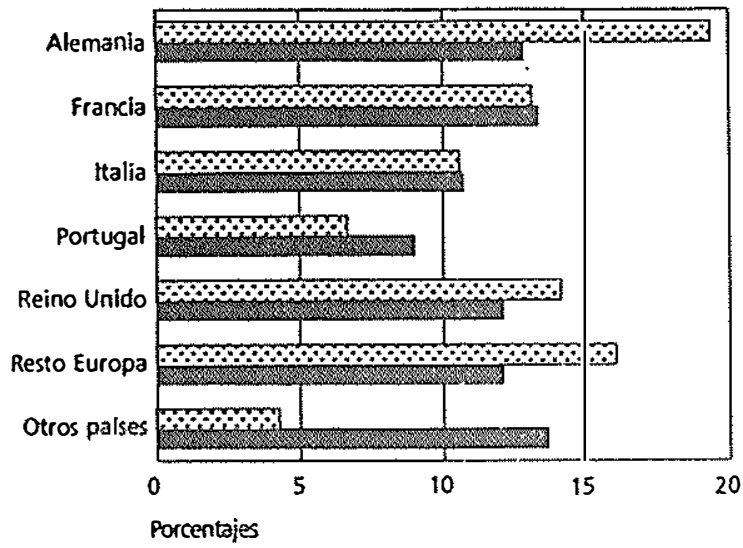

$\because$ Carretera Aeropuerto

\section{Turistas satisfechos e insatisfechos según diferentes conceptos.}

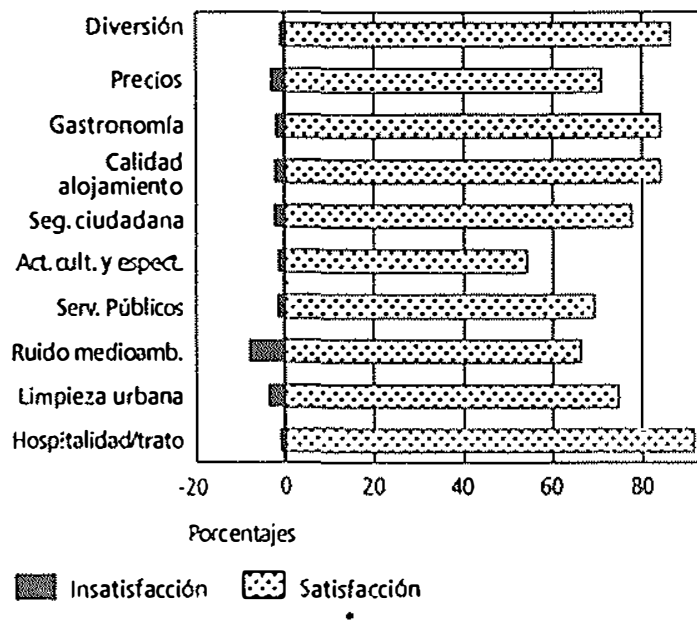

Un país acogedor pero... ruidoso
Andererseits

legen die Betriebe in der Aus- und Weiterbildung überwiegend Wert auf eine Zertifizierung der erworbenen Kenntnisse.

Diese Einschätzungen sind eine deutliche Kritik an dem
Rund 36\% der Unternehmen mit Fremdsprachenbedarf brauchen Englisch ständig und 45\% häufig. Jeder dritte Mitarbeiter benötigt Fremdsprachen (Tabelle 6).
Fremdsprachenunterricht der allgemeinbildenden Schulen, wo zuviel Wert gelegt wird auf das Üben von Grammatik, schriftlicher Sprachkompetenz und Literatur.

Der Schwerpunkt des Fremdsprachenbedarfs liegt eindeutig auf der mündlichen Kommunikationsfähigkeit und dem allgemeinen Wortschatz, die beide auch in der Muttersprache eine große Bedeutung haben.

Die weiteren Schwerpunkte bilden das Fachvokabular, das Hör- und das Leseverständnis. Erst dann folgen Grammatikkenntnisse, die schriftliche Fertigkeit und die Fähigkeit des Übersetzens.

Die meisten Betriebe sehen einerseits einen erheblichen Bedarf in der aktiven und allgemeinsprachlichen mündlichen Fremdsprachenkompetenz: Verhandlungen führen, telefonieren, Gespräche führen, verkaufen, usw.

\begin{tabular}{|c|c|c|c|c|}
\hline \multicolumn{5}{|c|}{$\begin{array}{l}\text { ESPANA } \\
\text { LLEGADAS DE TURISTAS PROCEDENTES DEL EXTRANJERO AL CONJUNTO DE LOS MEDIOS DE ALOJAMIENTOO }\end{array}$} \\
\hline PAIS & 1993 & 1994 & 1995 & 1996 \\
\hline Alemania & 3.322 .818 & 3.951 .430 & 4.276 .303 & 4.449 .743 \\
\hline Reino Unido & 2.758 .834 & 3.429 .881 & 3.542 .982 & 3.556 .189 \\
\hline Francia & 1.746 .150 & 1.882 .618 & 2.050 .712 & 2.084 .746 \\
\hline Italia & & 1.326 .128 & 1.303 .766 & 1.155 .554 \\
\hline Países Bajos & & 762.361 & 779.293 & 787.128 \\
\hline Bélgica & & 682.588 & 718.502 & 734.111 \\
\hline Portugal & & 442.527 & 491.390 & 507.281 \\
\hline Suiza & & 288.294 & 308.368 & 409.026 \\
\hline Suecia & & 214.523 & 197.685 & 216.221 \\
\hline Austria & & 142.389 & 265.306 & 180.199 \\
\hline Dinamarca & & 139.219 & 143.033 & 144.863 \\
\hline EEUU & & 802.595 & 835.383 & 919.786 \\
\hline Japón & & 380.516 & 477.710 & 616.852 \\
\hline
\end{tabular}


Die allgemeinbildenden Schulen haben demnach ihre Chancen bisher nicht wahrgenommen, die in der Wirtschaft wirklich wichtige aktive allgemeinsprachliche Kommunikationsfähigkeit in der Verwendung der Fremdsprache zu trainieren. Diese aktiven Fähigkeiten müssen länger und häufiger geschult werden als die rezeptiven Fähigkeiten (z.B. Leseverständnis), weil sie flüchtiger sind.

In diese "Marktlücke" hat das Pilot-Projekt zur "Sprachenqualifikation für Berufspraktiker im Tourismusbereich" eingeschlagen.

Die Universität Alicante entwickelt zur Zeit in Zusammenarbeit mit vier anderen EU-Ländern (Deutschland, Großbritannien, Frankreich und Italien) handlungsorientierte Sprachenqualifikationen für Mitarbeiter im Tourismusbereich.

Das Pilot-Projekt wurde im Rahmen des Gemeinschaftsprogramms Leonardo Da Vinci der Europäischen Kommission (GD XXII) im Jahr 1995 erstellt und beendet seine Konzipierung 1998.

Die Partner sind in Deutschland die Industrie- und Handelskammer zu Düsseldorf und die Wirtschaftsschule Düsseldorf, in Großbritannien das European Vocational College (London) -eine Berufsakademie der Deutsch-Britischen Industrie- und Handelskammer-, in Italien CESCOT -Centro de Sviluppo de Commercio, Turismo e Terziario (Rom)-, in Frankreich AFEC-Association Formation et Enseignement Continu (Bordeaux)- und in Spanien die Universität Alicante.

Sie haben zusammen eine Europäische Wirtschaftliche Interessenvereinigung gegründet. Die Vereinigung trägt folgenden Namen: "Institution zur Förderung europäischer Sprachenqualifikation für Berufspraktiker". Die Vereinigung hat zum Gegenstand die Förderung und die Schaffung von Sprachqualifikationen für Berufspraktiker im Tourismusbereich in Europa. Der Sitz befindet sich in Düsseldorf.

\begin{tabular}{|c|c|}
\hline FREMDSPRACHE & UND BETRIEBSGROßE \\
\hline $\begin{array}{l}\text { BETRIEBSGRÖßE } \\
\text { (Mitarbeiter) }\end{array}$ & FREMDSPRACHENDEBARF (Prozent) \\
\hline $1-49$ & 56.7 \\
\hline $50-99$ & 64.6 \\
\hline $100-499$ & 74.2 \\
\hline $500-999$ & 85.0 \\
\hline$\geq 1000$ & 92.9 \\
\hline $\begin{array}{l}\text { Quelle: Institu } \\
\text { Eindeutige Abhängigkeit des } \\
\text { festgestellt . Dementsprechet } \\
\text { höchsten Bedarf. }\end{array}$ & $\begin{array}{l}\text { Wirtschaft, Köln und IHK Düsseldorf, } 1998 \\
\text { enbedarfs von der Betriebsgröße } \\
\text { iebe mit über } 1.000 \text { Mitarbeitern den }\end{array}$ \\
\hline
\end{tabular}

\section{RANGFOLGE DER FREMDSPRACHEN OHNE UND MIT GEWICHTUNG NACH DER HÄUFIGKEIT}

\begin{tabular}{|c|c|}
\hline SPRACHENRANGFOLGE & NENNUNGEN EINFACH (Prozent) \\
\hline 1.Englisch & $445(97.4)$ \\
\hline 2.Französisch & $373(81.4)$ \\
\hline & \\
\hline 3.Spanisch & $216(47.1)$ \\
\hline 4.ttalienisch & $212(46.3)$ \\
\hline 5.Russisch & $111(24,2)$ \\
\hline 6. Niederlándisch & $86(48.8)$ \\
\hline 7.Tschechisch & $82(17.8)$ \\
\hline 8. Portugiesisch & $73(15.9)$ \\
\hline 9.Däniseh & $48(10.5)$ \\
\hline 10.Japanisch & $34(7,4)$ \\
\hline 11.Chinesisch & $29(6.3)$ \\
\hline 12.Polnisch & $18(3,9)$ \\
\hline 33.TGrklsch & $7(1.5)$ \\
\hline 14. Schwedisch & $5(1.1)$ \\
\hline 15. Ungarisch & $5(1.1)$ \\
\hline & \\
\hline 16.Bulgarisch & $2(0.4)$ \\
\hline 17.Griectisch & $2(0.4)$ \\
\hline 18. Koreanisch & $2(0.4)$ \\
\hline 19.Norwegisch & $2(0.4)$ \\
\hline 20.Serbokroatisch & $2(0.4)$ \\
\hline 21. Stowenisch & $2(0.4)$ \\
\hline 22.Arabisch & $1(0.2)$ \\
\hline $23 . F \mid$ nnisch & $1(0.2)$ \\
\hline 24.HIndI-Urdu & $1(0.2)$ \\
\hline 25.Indonesisch & $1(0.2)$ \\
\hline 26.Lettisch & $1(0.2)$ \\
\hline 27. Luxemburgisch & $1(0.2)$ \\
\hline 28.Rumänisch & $1(0.2)$ \\
\hline
\end{tabular}

Die Prüfungsordnung dient dem vergleichbaren Nachweis für Berufspraktiker im Tourismus, daß sie sich in berufstypischen Situationen fremdsprachlich angemessen und adressatengerecht verhalten können. Die Prüfung wird zunächst in den Partnerländern in allen Sprachen der Partnerländer angeboten; weitere Sprachen können nach Entscheidung der Partner jederzeit hinzukommen. Zielgruppen für diese Prüfung sind Mitarbeiter im Tourismus, wie zum Beispiel Fremdenführer sowie Mitarbeiter von Reisebüros, Reiseveranstaltern, Reedereien, Luftverkehrsunternehmen, Hotels, Restaurants, Verkehrsämtern, Museen und Messen.

Das Projekt wird nach entsprechender Verbreitung große Auswirkungen auf die Systeme und die Struktur der Berufsbildung haben, da einerseits dem Mündlichen eine herausragende Bedeutung zukommt (wie es übrigens auch der berufichen Praxis entspricht) und andererseits die Prüfungen europaweit harmonisiert werden können.

Tabelle 6

M.R.M.M.

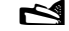
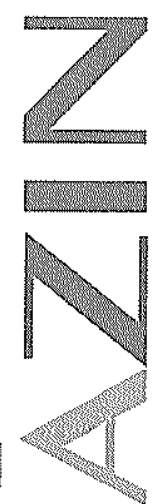